\title{
Energetic ion injection and formation of the storm-time symmetric ring current
}

\author{
L. Xie ${ }^{1}$, Z. Y. Pu ${ }^{1}$, X. Z. Zhou ${ }^{1}$, S. Y. Fu ${ }^{1}$, Q.-G. Zong ${ }^{2}$, and M. H. Hong ${ }^{3}$ \\ ${ }^{1}$ School of Earth and Space Sciences, Peking University, Beijing, 100871, China \\ ${ }^{2}$ Center for Space Physics, Boston University, Boston, 02215, USA \\ ${ }^{3}$ Institute of Geology and Geophysics, Chinese Academy of Science, 100029, China
}

Received: 22 March 2006 - Revised: 7 November 2006 - Accepted: 24 November 2006 - Published: 21 December 2006

\begin{abstract}
An extensive study of ring current injection and intensification of the storm-time ring current is conducted with three-dimensional (3-D) test particle trajectory calculations (TPTCs). The TPTCs reveal more accurately the process of ring current injection, with the main results being the following: (1) an intense convection electric field can effectively energize and inject plasma sheet particles into the ring current region within $1-3 \mathrm{~h}$. (2) Injected ions often follow chaotic trajectories in non-adiabatic regions, which may have implications in storm and ring current physics. (3) The shielding electric field, which arises as a consequence of enhanced convection and co-exists with the injection and convection electric field, may cause the original open trajectories of injected ions with higher energy to change into closed ones, thus playing a role in the formation of the symmetric ring current.
\end{abstract}

Keywords. Magnetospheric physics (Electric fields; Plasma convection; Storms and substorms)u

\section{Introduction}

Most intense magnetic storms are a response of the magnetosphere to the passage of coronal mass ejections from the Sun. They are initiated when enhanced energy transferred from the solar wind into the magnetosphere leads to intensification of the ring current (Tsurutani et al., 1997). The ring current mainly consists of $10-200 \mathrm{keV}$ ions and electrons that drift azimuthally around the Earth or pass over the inner magnetosphere at radial distances of about 2-7 Earth radii $\left(R_{E}\right)$. The main enhancement of the storm-time ring current occurs at distances $L<4$. Two kinds of electric fields have been considered as being the fundamental cause of the main phase injection of ring current particles: the induced electric field

Correspondence to: $\mathrm{Z}$. Y. Pu

(zypu@pku.edu.cn) associated with substorm dipolarization of the geomagnetic field and the enhanced convection electric field $E_{C}$ driven by a strong negative z-component of the interplanetary magnetic field (IMF). It has been shown that the dipolarization induced electric fields can readily inject particles from the inner plasma sheet to the near synchronous altitudes; however, they cannot displace particles at significant distances below $L=4$. This indicates that substorms are not fundamental to the injection of ring current particles to $L<4$ (Tsurutani et al., 1997). On the other hand, it has been found that there is a one-to-one relation between intense $\left(D_{s t}<-100 \mathrm{nT}\right)$ storms and large negative IMF $B_{z}(<-10 \mathrm{nT})$ that lasts for at least $3 \mathrm{~h}$ (Kamide et al., 1997). Numerical calculations show that the intense magnetospheric convection electric field $E_{C}$ can effectively energize (within 2 to $3 \mathrm{~h}$ ) charged particles while they are injected from the magnetotail to the ring current region $(L \sim 3)$. The required electric fields were observed by the CRRES satellite to occur in the $L=2-4$ region at times of ring current injection (Wygant et al., 1998). Therefore, it is now commonly accepted that the enhanced $E_{C}$ is mainly responsible for the ring-current particle injection and the formation of storm-time ring current (Tsurutani et al., 1997).

Charged particles in the magnetosphere have two types of drift paths: a closed trajectory around the Earth and an open trajectory traveling from the magnetotail to the magnetopause. In the conventional ring current pictiure, the "symmetric" ring current consists of trapped particles with closed orbit, while the particles with open convection paths create an "asymmetric" or partial ring current (Le et al., 2004). The $D_{s t}$ index and SYM-H index are reliable indicators of the symmetric ring current strength, while the ASYM-H index measures the asymmetric ring current. The conventional picture of the ring current should be understood as an approximation of the first order. In fact, the closed-trajectory of ions may be spatially asymmetric, hence also contributing to the ASYM-H (Liemohn et al., 2001). A separatrix boundary called the Alfvén layer exists between the open and closed

Published by Copernicus GmbH on behalf of the European Geosciences Union. 
trajectories (Wolf, 1995; Xie et al., 2002). These two types of trajectories are un-transformable to each other when the electric and magnetic fields are constant in time (Lyons and Williams, 1984; McPherron, 1991). Injection of charged particles caused by $E_{C}$ does not directly make a contribution to the formation of the symmetric current. The process of the ring current particle injection during the main phase of the storms and the formation of the symmetric ring current demands detailed studies.

It has been suggested that the time variations in the convection electric field can make the separatrix between the open and closed trajectories of higher- $\mu$ particles indistinct. This may cause the charged particles convected from the tail to diffuse across the boundary and to be trapped on a closed-loop trajectory (Rowland and Wygant, 1998). Chen et al. $(1994,1997)$ made a numerical modeling of a great storm whose average enhancement in the cross-tail potential drop $\Phi_{C}$ is $180 \mathrm{kV}$. They assumed the following two conditions: Eq. (1) $\Phi_{C}$ has a standing fluctuation lasting for $12 \mathrm{~h}$ with a cycle being $20 \mathrm{~min}$ and peaks reaching $300-400 \mathrm{kV}$; Eq. (2) fluctuation lasts for $3 \mathrm{~h}$, then $\Phi_{C}$ remains constant for another $6 \mathrm{~h}$. The modeling showed that most ions injected from the nightside plasma sheet with energy of $10-70 \mathrm{keV}$ at $L \sim 3$ were transported along open drift paths. Conversely, the transport of particles with energies greater than $150 \mathrm{keV}$ at $L \sim 3$ was appropriately detailed as radial diffusion across closed drift trajectories. In Eq. (2) the influence of the fluctuations on the ion trajectories is found to be very small. While in Eq. (1) the total energy of the ring current particles was nearly a factor of two larger than that in Eq. (2) (Tsurutani et al., 1997; Chen et al., 1994). These results make scientists believe that the formation of the closed ring current relies on the presence of storm-associated fluctuations in the convection electric field. Nevertheless, investigations of the correlation between the interplanetary electric field and Dst indices during the storm time imply that the Earth's $E_{C}$ does not always possess the long-lasting fluctuations required in Eq. (1) (Zhang et al., 2001). Data from CRRES observations indicated that the storm-time electric fields show large fluctuations (Wygant et al., 1998), but the oscillation peaks are considerably less than the $300-400 \mathrm{kV}$ used by Chen et al. $(1994,1997)$ in simulations. Therefore, it is reasonable to consider that the fluctuation of $E_{c}$ is probably not the only mechanism contibuting to the formation of the closed ring current.

It was discovered long ago that the inner edge of the plasma sheet tends to shield the inner magnetosphere from the convection electric field (Kavanagh et al., 1968; Schield et al., 1969). In addition, while injecting inward by $E_{C}$ from the plasma sheet, electrons and ions tend to drift dawnward and duskward, respectively. This may also lead to a "shielding electric field" $E_{S}$ that opposes to the overall dawn to dusk electric field. The presence of $E_{S}$ makes the total dawn to dusk electric field weaker than that without $E_{S}$, and even negligibly small in the low L-region. In this paper, we pro- pose that the shielding of the inner magnetosphere from the $E_{C}$ may cause a change in an open convection drift path into a closed-loop trajectory, and hence play an important role in the processes of the storm-time injection and the formation of the symmetric ring current.

In the present study, we apply three-dimensional (3-D) test particle trajectory calculations (TPTCs) to investigate the particle injection from the inner plasma sheet and the formation of the symmetric ring current. We focus on the drifting paths of energetic oxygen ions whose abundance and energy density in the ring current are extraordinarily high in the main phase of intense magnetic storms (Daglis et al., 1997; Fu et al., 2001). A semi-quantitative and time-independent shielding electric field model based on observations and physics analyses is adopted. In this paper we will first discuss the appearance of the shielding electric field and its influence on the convection path of injecting oxygen ions, and then introduce the TPTCs method, and the magnetic and electric field models used in the calculations. In Sect. 4 we present a part of the preliminary results. In Sect. 5 we make a short discussion and a brief summary.

\section{Formation and effect of the shielding electric field}

The drift motion of charged particles in the magnetosphere is broken down into three components: the magnetic field gradient drift $v_{G}=W_{\perp} B \times \nabla B / q B^{3}$, the field line curvature drift $v_{c}=2 W_{/ /} R \times B / q B^{2} R^{2}$ and the electric field drift $v_{E}=E \times B / B$, where $W_{\perp}$ and $W_{/ /}$are the particle kinetic energy perpendicular and parallel to the magnetic field, respectively, $q$ is the particle charge, $\nabla B$ represents the magnetic field gradient, $R$ denotes the curvature radius of the magnetic field line. When energetic particles are injected by $E_{C}$ from the magnetotail to the inner magnetosphere, under the action of the co-existing gradient and curvature drifts, most ions and electrons tend to drift duskward and dawnward of the Earth, respectively. As a whole, electrons drift closer to the Earth on the dawnside than ions. In the same way, ions drift closer to the Earth on the duskside than electrons. Figure 1 shows the calculated drift trajectories of ions with $90^{\circ}$ pitch angle in the equatorial plane calculated by 3-D TPTCs. The asymmetrical, noncircular drift paths for ions and electrons lead to a charged-separation layer near the dipole where particles of the opposite sign are separated by the drift motion. Schield et al. (1969) called this charge separation layer the Alfvén layer. Thus the dawnside inner edge and duskside inner edge tend to charge up negatively and positively, respectively. Figure 2 shows the sketch of the drift paths of ions and electrons moving in a dipole magnetic field with a uniform electric field. In this illustration, a net positive charge will accumulate on the duskside of the Earth and a negative charged will be gathered on the dawnside.

To keep charge from accumulation indefinitely, fieldaligned currents flow from the magnetosphere to the 


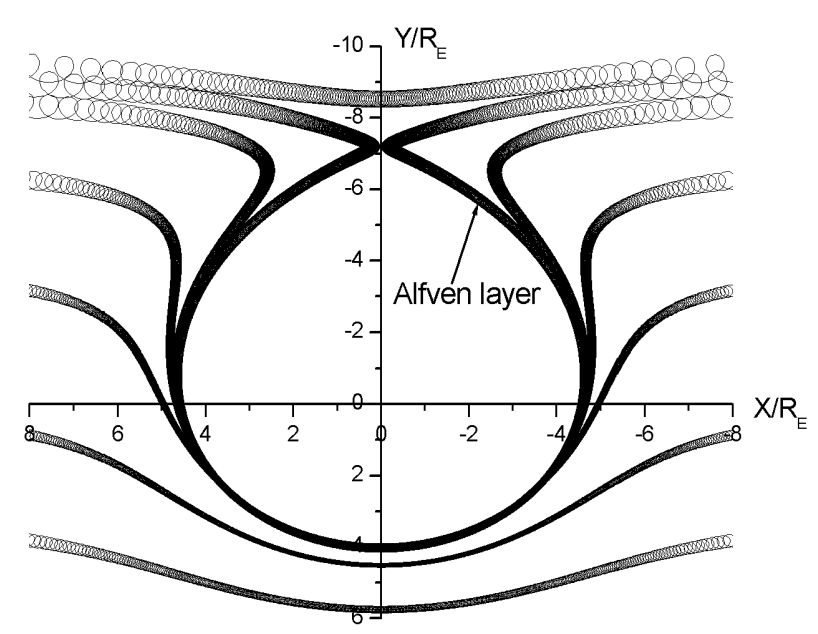

Fig. 1. Drift trajectories of ions from different positions in the equatorial plane.

conducting ionosphere on the duskside, and from the ionosphere to the magnetosphere on the dawnside (Wolf et al., 1982). As a result of this charge separation, a shielding electric field $E_{S}$ occurs whose direction is opposite to the overall dawn to dusk $E_{C}$. The shielding configuration is shown schematically in Fig. 3. In addition to the energetic particles, lower energy particles also have their Alfvén layers, which are closer to the Earth than particles with higher energies. The convection flow of the plasma sheet with lower energy population may yield a velocity shear in the lower L-region. Based on Sato (1982), this flow shear also produces positive and negative net charges on the duskside and dawnside of the inner magnetosphere, respectively, thus creating an additional part of the shielding electric field. This is an alternative mechanism responsible for the buildup of the region-2 field-aligned current (Hasegawa and Sato, 1979). In the presence of $E_{S}$, the total dawn to dusk electric field is weaker than that without $E_{S}$. The shielding electric field may even shield some local areas of the low-L region $(L<5)$ completely from the $E_{C}$. It is clear that $E_{S}$ arises as a consequence of enhanced convection and co-exists with the persistence of injection and $E_{C}$. The distribution and evolution of $E_{S}$ are determined by the self-consistent interaction between injected particles and the convection electric field, and vary along with the variations of $E_{C}$. The concept of the shielding electric field was first proposed by Kavanagh et al. (1968). Thereafter Wolf et al. (1982), through a number of computer simulation tests, confirmed the occurrence of $E_{S}$ in the inner magnetosphere accompanied by an intense $E_{C}$. CRRES further observed the effect of $E_{S}$ in the low-L region and its relationship to the geomagnetic activity (Rowland and Wygant, 1998). The magnitude of $\Phi$ is decreased with increasing distance to the Earth. More details about the magnetospheric shielding can be founded in the literature.

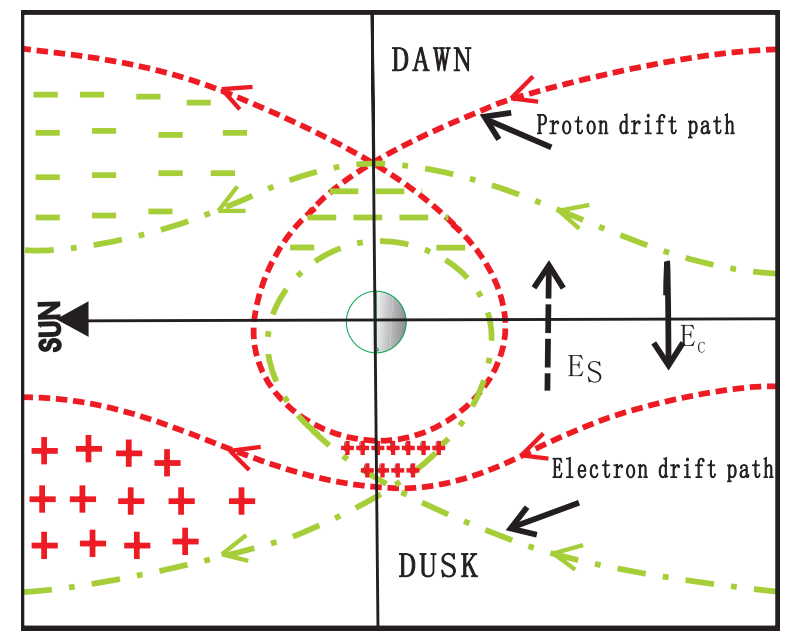

Fig. 2. Sketch of drift paths of ions and electrons moving in a dipole magnetic field with a uniform electric field.

Recently, a few scientists have realized that $E_{S}$ may probably be a factor in the formation of the ring current (Fok et al., 2001; Ebihara et al., 2004). The gradient and curvature drift under the inner magnetospheric condition tends to cause particles to move around the Earth. They therefore are advantageous to the formation of the closed orbit. The higher the particle energy is, the more intense this effect becomes. On the other hand, the open trajectories originate from the $E_{C} \times B$ drift. The larger the magnitude of $E_{C}$ is, the stronger the sunward injection becomes. Suppose that in the early main phase of a storm, energetic particles are injected from the plasma sheet into the inner magnetosphere along open trajectories. If there were no $E_{S}$, the injection paths would remain open forever. Once the shielding field is created, the sunward drift will be weakened, the relative importance of the gradient and curvature drift will be increased. This may cause the original open trajectories (in the case without $E_{S}$ ) to change into closed ones, in particular for energetic particles with higher energies. To summarize this section in short: the storm-time ring currents consist of both the asymmetric and symmetric ring current. The former should first enhance in the early main phase, the latter reinforces in the late time. The enhancement of the symmetric ring current manifests the intrinsic consequence of particle injection, which should take place prominently after the increase in the asymmetric ring current, and is composed of the energetic ions with higher energies. Calculations made in this paper confirm this conjecture, suggesting that the shielding electric field plays an important role in the ring current particle injection and the formation of the symmetric ring current. 

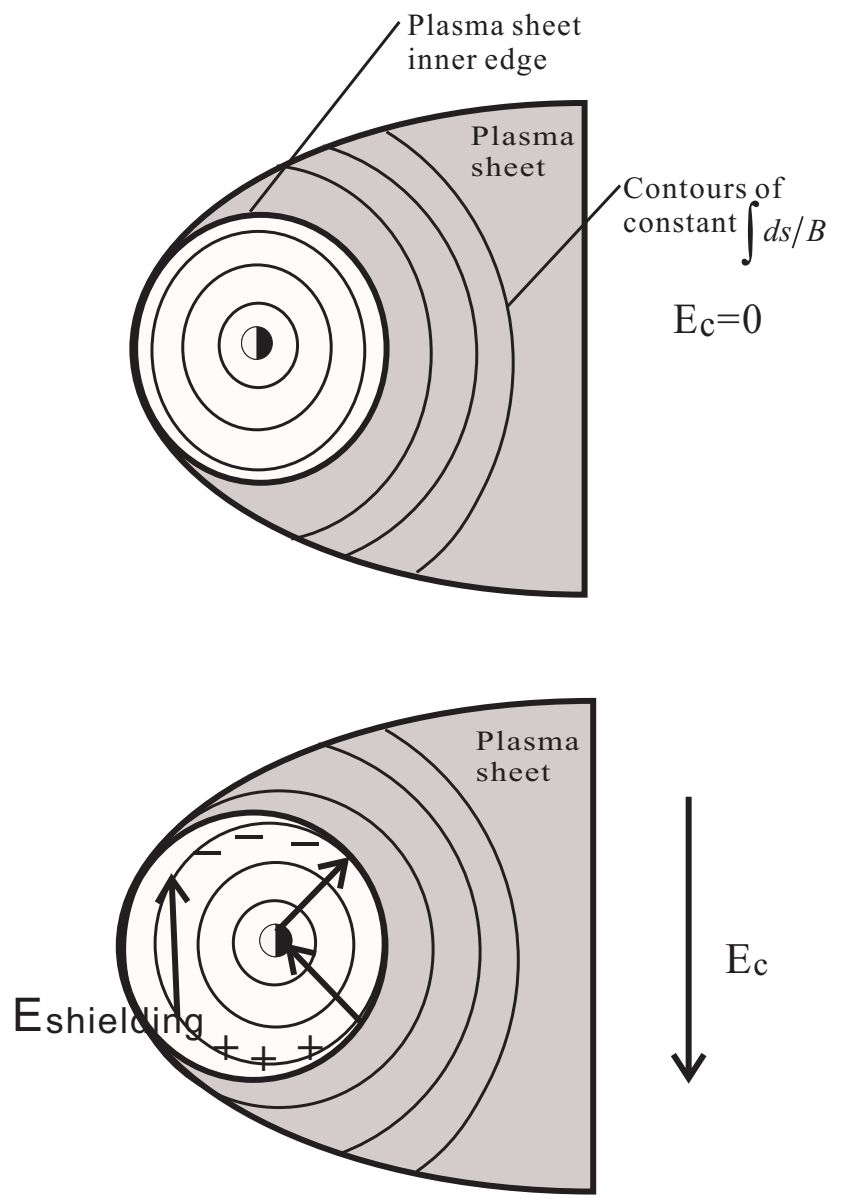

Fig. 3. Illustration of the formation of shielding electric field.

\section{3-D TPTC and magnetic and electric field models}

\subsection{3 -D TPTC}

Charged particle motion in the magnetosphere is divided into three components: the gyro motion, bounce motion and drift motion. The TPTC method exactly tracks the full motion of a charged particle which reads

$\frac{d p}{d t}=q \times\left(E+\frac{d r}{d t} \times B\right)$.

In addition to Eq. (1), we have

$p=m_{0} \gamma d r / d t$

where

$\gamma=\sqrt{1-v^{2} / c^{2}}$.

$m_{0}$ and $p$ represent the rest mass and relativistic momentum of the particle, respectively. At $t=0$ the test particle is situated in the source region. The initial equatorial pitch angle of the test particle can be assigned an arbitrary value outside the loss cone.

\subsection{Magnetic field model}

The T96 magnetic field model (Tsyganenko et al., 1996) is used in calculations, which is a semiempirical global model based on a large number of satellite observations (IMP, HEOS, ISEE, POLAR, Geotail, etc.). It incorporates the official International Geomagnetic Reference Field (IGRF) model as the Earth's internal field and includes the contributions from external magnetospheric sources: ring current, magnetotail current system, magnetopause currents and large-scale system of field-aligned currents. The Earth's dipole magnetic field model is also adopted in some calculation runs for comparison.

\subsection{Electric field models}

\subsubsection{Convection electric field models}

Two main sources of magnetospheric electric fields are considered: the dawn-to-dusk $E_{c}$ field associated with the enhanced magnetospheric convection and the corotation electric field $\left(E_{c o}\right)$ related to the rotation of the Earth along its spin axis. Besides, the shielding electric field in conjunction with injection and enhanced convection is also included, as mentioned before.

Three convection electric field models are adopted in this paper:

- The uniform convection field model:

$\Phi_{C}=-E_{0} r \sin (\phi)$,

where $\Phi_{C}$ represents the cross-tail potential of the convection electric field and $\Phi_{C}=0$ at 12:00 MLT, $\phi$ denotes the azimuthal angle in the GSM system, $r$ is the geocentric distance, $E_{0}$ represents the uniform convection electric field.

- The Volland-Stern model (Volland, 1978):

$\Phi_{C}=-K r^{2} \sin (\phi)$.

$K=\frac{0.045}{\left(1-0.159 K_{P}+0.093 K_{P}^{2}\right)^{3}} k V / R_{E}^{2}$,

where $K$ depends only on the $K_{P}$ index (Maynard and Chen, 1975), $\Phi_{C}$ is measured in $\mathrm{kV}$ and $R_{E}$ denotes the Earth's radius.

- The Heppner-Maynard-Rich model (Heppner et al., 1987):

The Heppner-Maynard-Rich (MHR) electric field model is constructed based on Polar-Orbiting Geophysical Observatory 6 (OGO 6) and Dynamics Explorer 2 (DE 2) electric field measurements (Heppner et al., 1987). It provides the electric potential and field poleward of $60^{\circ}$ geomagnetic latitude. Readers are referred to the original reference for details of the MHR model. 

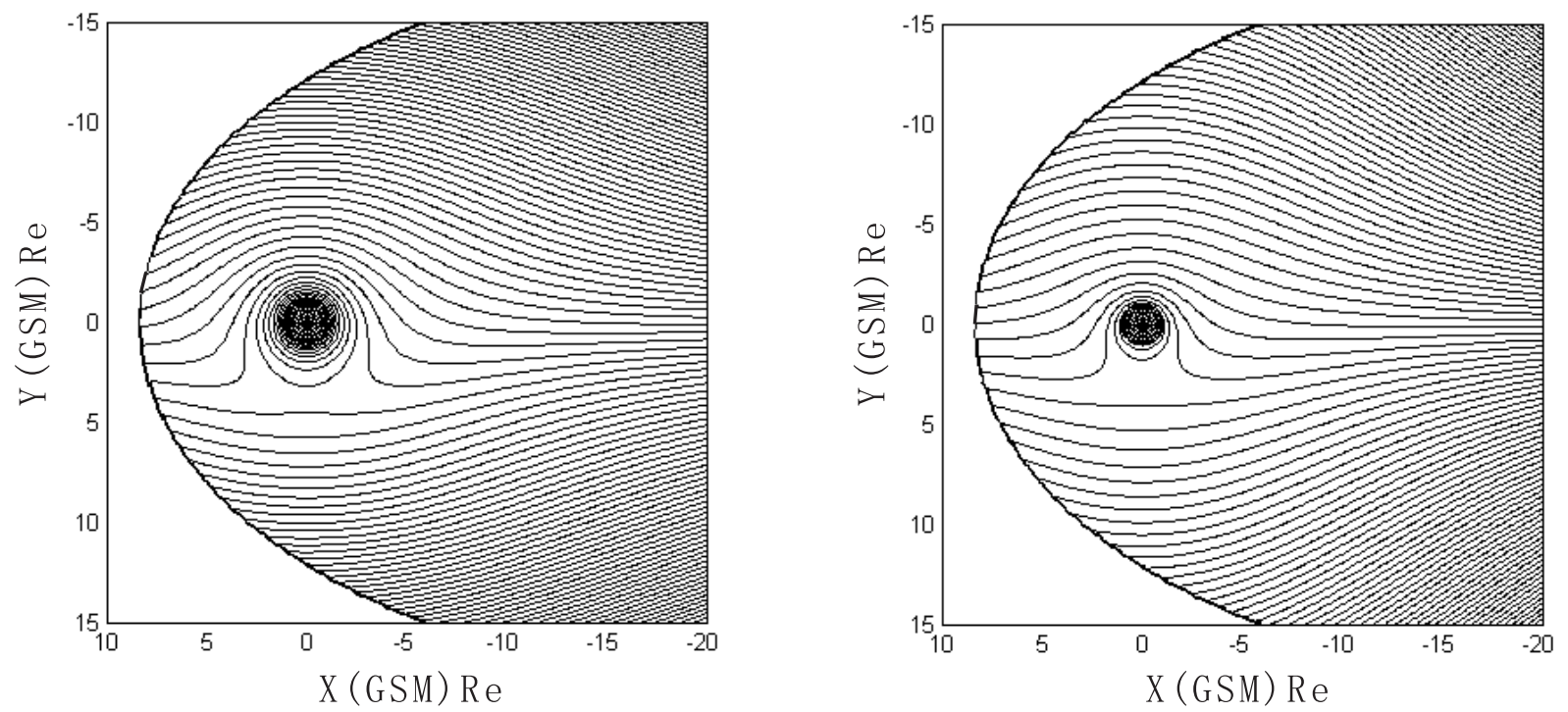

Fig. 4. Equipotential contours of the total electric field. Left: Sum of uniform convection electric field and corotation electric field. Right: Sum of convection, shielding and coratation electric field.

\subsubsection{Corotation electric field models}

If the magnetic field in the near-Earth region is approximately represented by a dipolar field with its moment antiparallel to the Earth's rotation axis, the co-rotation electric field can be expressed as (Lyons and Williams, 1984)

$\Phi_{c o}=-\omega_{E} R_{E}^{3} B_{0} / r$

where $\omega_{E}$ is the angular velocity of the Earth and $B_{0}$ is the field magnitude on the Earth's surface at the equator.

\subsubsection{Shielding electric field model}

For simplicity we choose the time when the tilt angle is minimum near the equinoxes, so that GSM $Z \approx 0$ in the equatorial plane where the magnetic field is minimum along the field lines. The following steady-state shielding electric field model is adopted at the equatorial plane:

$\Phi_{s}(x, y)=A+B x^{2} y$,

where $x$ and $y$ are coordinates of the GSM system, $A$ and $B$ are constant. This shielding electric field is not selfconsistent. Suppose that during the later main phase of the intense magnetic storms the inner magnetosphere region $-8 R_{E}<x<8 R_{E}$ is partially shielded from the convection electric field, we obtain $A=8 \mathrm{kV}$ and $B=0.02 \mathrm{kV}$.

While using these models in the calculations, we regard the field lines as equipotential lines by assuming an infinitively large conductivity along the field lines. For particles mirroring at higher latitudes, we trace the field lines from the equator to off-equatorial points, or vice versa, to obtain the corresponding potential and electric fields inside the magnetosphere.
Figure 4 shows the equipotential contours of the total electric field in the equatorial plane without (left) or with (right) the shielding electric field. In these figures, the convection electric filed model (Eq. 5) and corotation electric field (Eq. 7) have been used. One can clearly see that the presence of the shielding electric field (Eq. 8) makes overall dawn to dusk electric field apparently weaker than without the shielding effect. It should be mentioned that the evolution of $E_{C}$, $E_{S}$, particle injection and the partial and symmetric ring current is a self-consistent coupled dynamical process. Carrying out TPTCs under steady-state conditions can only reveal some key features of physics, but not the real and whole process. The purpose of adopting the shielding field model (Eq. 8) is just to illustrate qualitatively the important role that $E_{S}$ plays in the storm-time injection and formation of the symmetric ring current, but not to obtain a strictly quantitative result.

\section{Calculation results and discussions}

Two sources of the oxygen ion population have been considered: energetic $\mathrm{O}^{+}$, with a few tens of $\mathrm{keV}$, originally located at $\mathrm{L} \leqq 8$ or $L \sim 15$ and the thermal ions $(\leqq 10 \mathrm{keV})$, upflowing from the Earth's ionosphere. The initial conditions for the test particles at $t=0$ are set as follows: $E=5 \sim 20 \mathrm{keV}$, $x=-7 \sim-15 R_{E}$ and $y=-2 \sim-8 R_{E}$. The cross-tail convection electric potential is chosen to be $150 \mathrm{kV}$.

\subsection{Injection and energization of ring current $\mathrm{O}^{+}$ions}

The top diagram of Fig. 5 plots the trajectories of $\mathrm{O}^{+}$initially situated at $x=-8 R_{E}, y=-2 R_{E}$ and $z=0$ with energy 

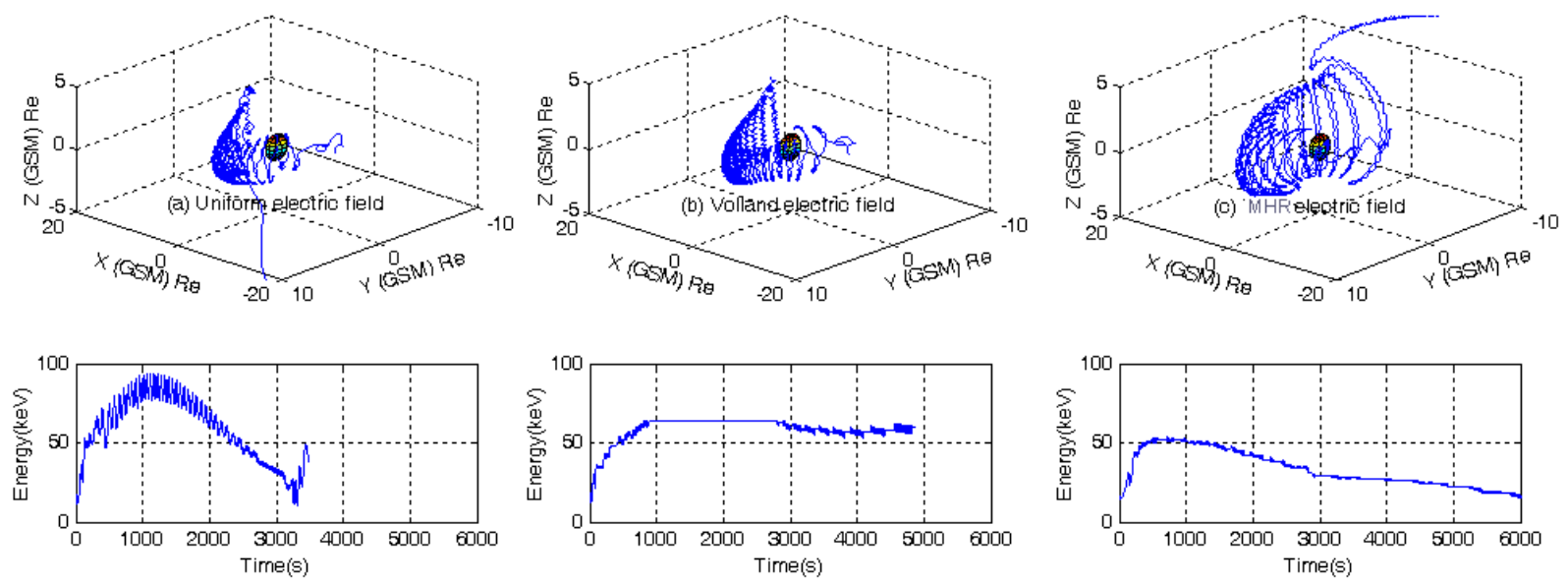

(a)

(b)

(c)

Fig. 5. Three drift trajectories of $15 \mathrm{keV} \mathrm{O}^{+}$with an equatorial pitch angle of $60^{\circ}$ initially situated at $x=-8 R_{E}, y=-2 R_{E}$ and $z=0$ and the energization process for the case corresponding to the Uniform, Volland and MHR electric fields in the T96 $D_{s t}=-100 \mathrm{nT}$ magnetic field model.
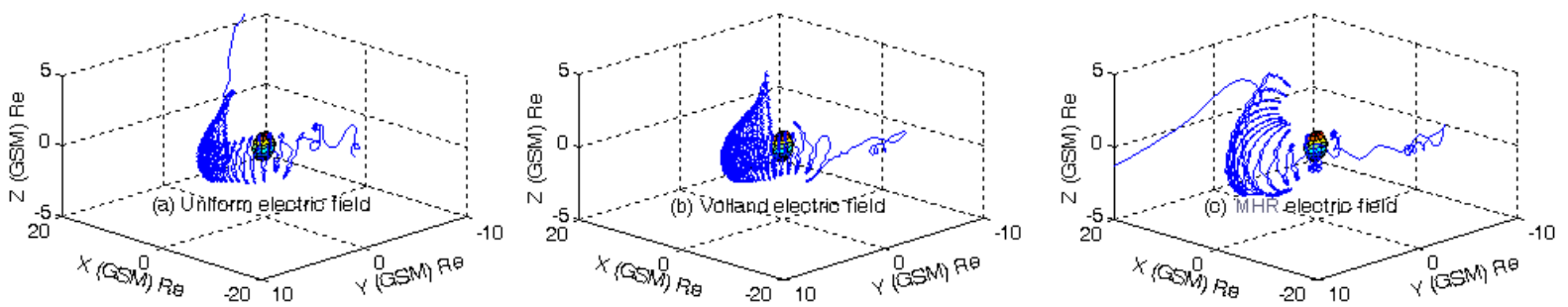

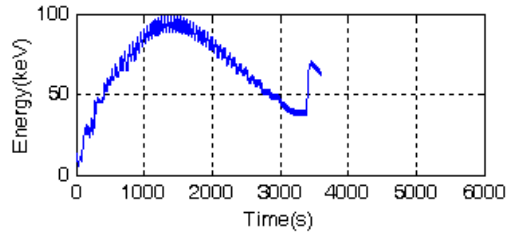

(a)

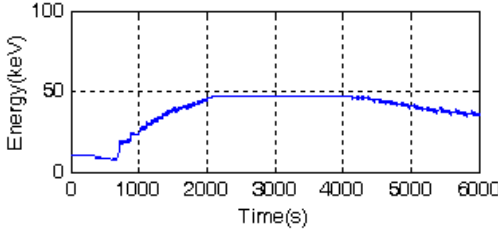

(b)

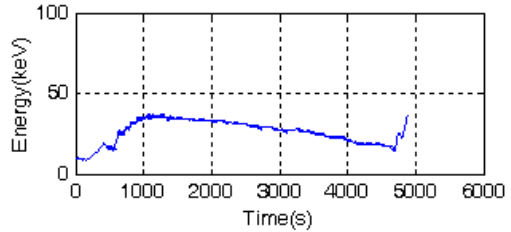

(c)

Fig. 6. Three drift trajectories of $10 \mathrm{keV} \mathrm{O}^{+}$with an equatorial pitch angle of $60^{\circ}$ initially situated at $x=-12 R_{E}, y=-3 R_{E}$ and $z=0$ and the energization process for the case corresponding to the Uniform, Volland and MHR electric fields in the T96 with $D_{s t}=-100 \mathrm{nT}$ magnetic field model.

$E=15 \mathrm{keV}$ and pitch angle $\alpha=70^{\circ}$. The electric field models used in (a), (b) and (c) are the uniform, Volland-Stern, and HMR models, respectively. The T96 magnetic field model with $D_{s t}=-100 \mathrm{nT}$, IMF $B_{y}=0, B_{z}=-10 \mathrm{nT}$ and solar wind pressure $P_{s w}=10 \mathrm{nPa}$ is used in drawing all these trajectories. The bottom diagram of Fig. 5 illustrates the corresponding energization process via drift time. Figure 6 plots the drift paths of $\mathrm{O}^{+}$initially situated at $x=-12 R_{E}, y=-3 R_{E}$, and $z=0$ with energy $E=10 \mathrm{keV}$ and pitch angle $\alpha=60^{\circ}$. The electric field models and magnetic field model used are the same as those in Fig. 5. It is shown in Figs. 5 and 6 that particles initially located in the near-Earth tail can be effectively energized and injected into the inner magnetosphere within
1-3 $\mathrm{h}$ and that all trajectories of these particles are open. For the case of uniform $E_{C}$, the injection and energization time can even be as short as a few tens of minutes. Because of the page limitation, we cannot show all interesting plots in the paper. Nevertheless, the following conclusion can be drawn: the intense convection electric field can effectively energize the injected particles; the stronger the convection electric field/the closer the inner edge of the plasma sheet, the shorter the injection time. These results are in agreement with the fact that intense storms, in general, occur in the cases when a strong $E_{C}$ lasts longer than $3 \mathrm{~h}$ and the decreasing time of the main phase in some storms is only several tens of minutes to $1 \mathrm{~h}$. 


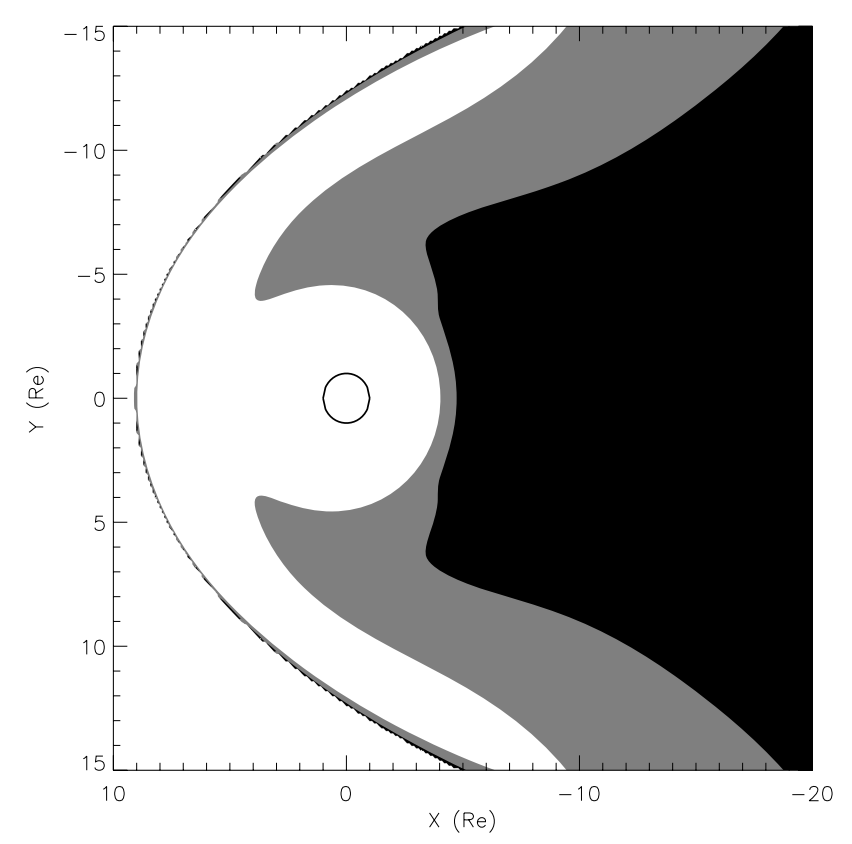

Fig. 7. The spatial distribution of $\kappa<3$ for $20 \mathrm{keV} \mathrm{O}^{+}$(gray and black) and protons (black) in the equatorial plane of the magnetosphere based on T96 model with $D_{s t}=-100 \mathrm{nT}$ and Solar Wind Pressure $=10 \mathrm{nPa}$.

\subsection{Chaotic injection trajectories}

The present understanding of the ring current injection is mostly based on the modeling studies in which the magnetic field is usually assumed to be the dipole field. With this approximation the drift trajectory of an injection particle generally manifests a regular path. However, it is found in our calculations that when a more realistic magnetic field model (such as T96) is adopted, the storm-time injection often possesses disordered orbits in certain spatial regions. We refer to these type of orbits as the chaotic trajectories, which appear under the condition that the gyro-radii of the charge particles are comparable to the curvature radii of the field lines at the equator (Speiser et al., 1991), where particles lose their adiabaticity. When particles are passing over the equatorial plane, they will miss the field line that they originally circle around and stochastically change their pitch angles. This happens to both Oxygen ions and protons. As defined by $\sqrt{R_{\text {cur }} / R_{g}}\left(R_{\text {cur }}\right.$ is the curvature radii of the field lines, $R_{g}$ is the gyro-radii of charge particles), the values of $\kappa$ greater than 3 indicate the adiabatic motion of the certain particle. In the regions with $\kappa$ less than 3 , the magnetic moment would no longer conserve, indicating the appearance of chaotic trajectories. Figure 7 shows the spatial distributions of $\kappa<3$ for $\mathrm{O}^{+}$and protons with initial energy being $20 \mathrm{keV}$ in the equatorial plane of the magnetosphere based on the T96 model. The black region is the chaotic region $(\kappa<3)$ for both protons and Oxygen ions, while the gray one is only chaotic for Oxy-

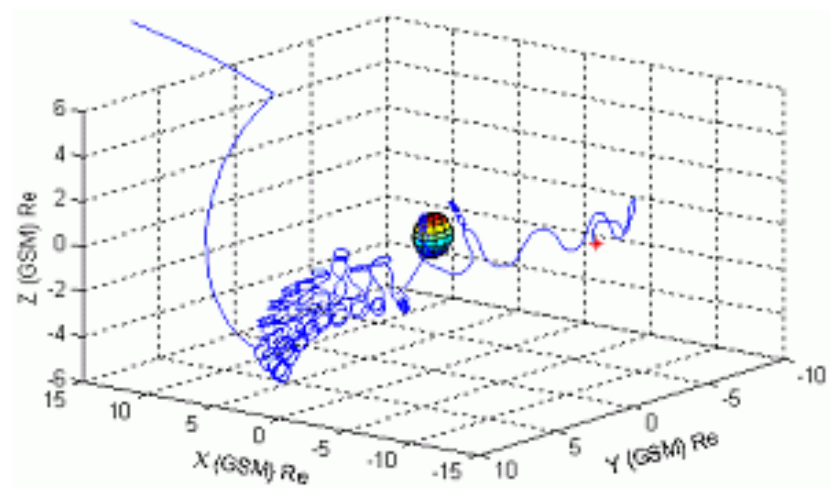

Fig. 8. A chaotic injection trajectory of an $20 \mathrm{keV} \mathrm{O}^{+}$with equatorial pitch angle of $60^{\circ}$ initially located at $x=-8 R_{E}, y=-2 R_{E}$, and $z=0$ in T96 with $D_{s t}=-100 \mathrm{nT}$ magnetic field model and HMR electric model.

gen ions. It is seen that the chaotic trajectories of $\mathrm{O}^{+}$can be much closer to the Earth than protons. Thus, the injecting $\mathrm{O}^{+}$ will more easily be trapped in the inner magnetopshere under the action of fluctuations and the shielding electric field.

In the realistic geomagnetic field the minimum $B$ on each field line is not exactly located on the equatorial plane, hence the pitch angle of an originally equatorially mirroring particle can also stochastically vary in the non-adiabatic region, and then the particle begins to bounce along the field lines. Figure 8 illustrates a chaotic injection trajectory of an $\mathrm{O}^{+}$ particle initially located at $x=-8 R_{E}, y=-2 R_{E}$, and $z=0$ with energy $E=20 \mathrm{keV}$ and pitch angle $\alpha=60^{\circ}$. T96 with $D_{s t}=-100 \mathrm{nT}$ and the HMR model are used in calculations. It is shown that the stochastic changing of the pitch angle may cause the particle to move, approaching the Earth along the field lines, and even down to the ionosphere. Chaotic motion may possibly displace particles across the Alfvén layer, changing an open drift path into a closed path circling the Earth. This may also make a contribution to the enhancement of the symmetric ring current. We will make a detailed study regarding this topic in a future paper.

\subsection{Effect of the shielding electric field}

The primary result of this paper is the fact the 3-D TPTCs reveal that the shielding electric field may play an important role in the development of the storm-time ring current. Figure 9 illustrates the different injection paths for an equatorially mirroring $\mathrm{O}^{+}$with or without the action of the shielding electric field Eq. (7). At $t=0$ the Oxygen ion is located at $x=-8 R_{E}, y=-3 R_{E}$ and $z=0$ with the initial energy $E=15 \mathrm{keV}$ for the upper panel and $E=5 \mathrm{keV}$ for the lower panel, respectively. The Volland and Stern electric field model (Eq. 4) and the Earth's dipolar magnetic field are adopted in the calculations. It is seen in the upper panel that the original open trajectory of $15 \mathrm{keV} \mathrm{O}^{+}$with vanishing $E_{s}$ turns closed when the shielding field (Eq. 4) is added. 

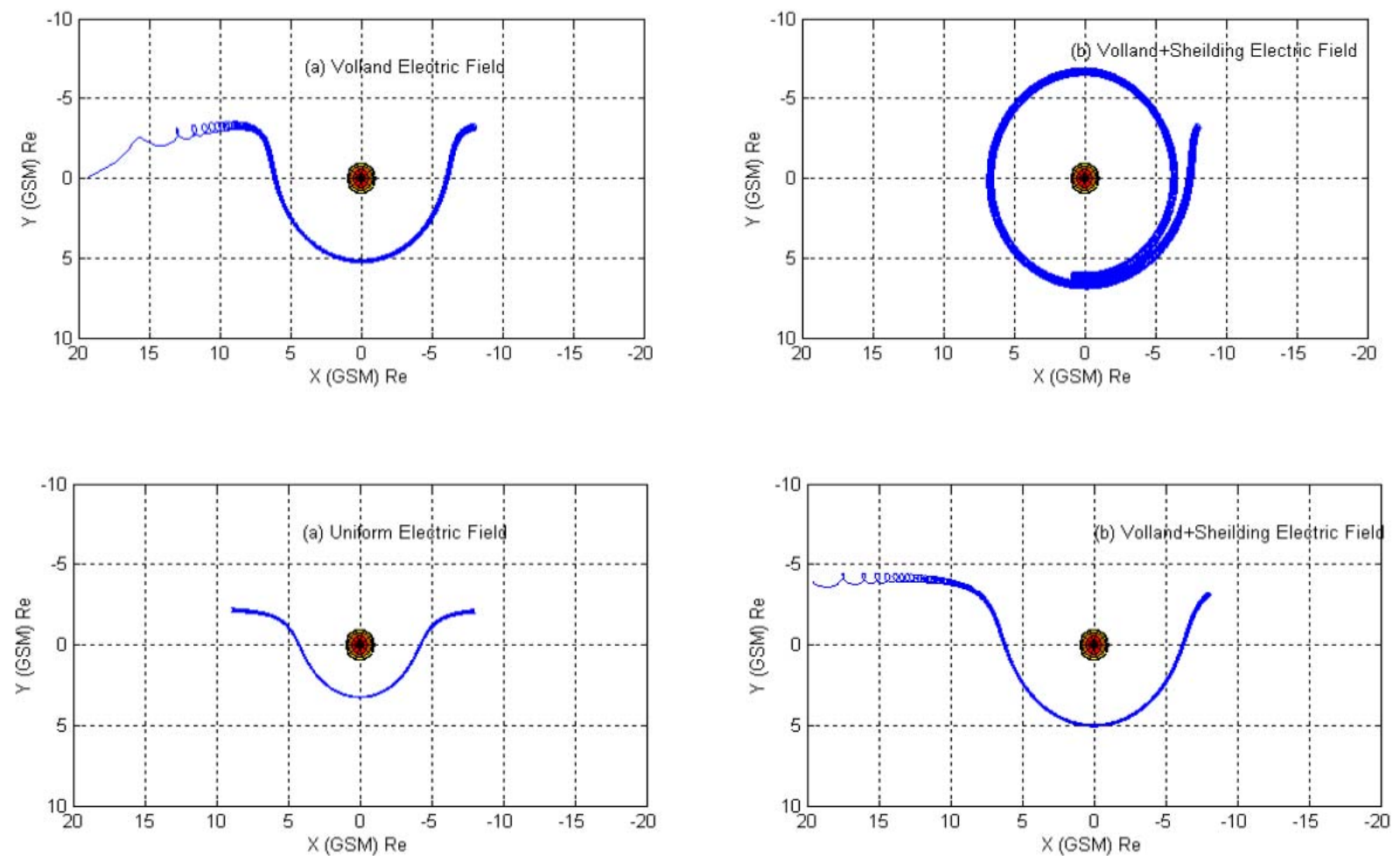

Fig. 9. Different injection paths for an equatorially mirroring $\mathrm{O}^{+}$initially situated at $x=-8 R_{E}, y=-3 R_{E}$ and $z=0$ with or without the action of the shielding electric field. The initial energy is $E=15 \mathrm{keV}$ for the upper panel and $E=5 \mathrm{keV}$ for the lower panel.

(a)

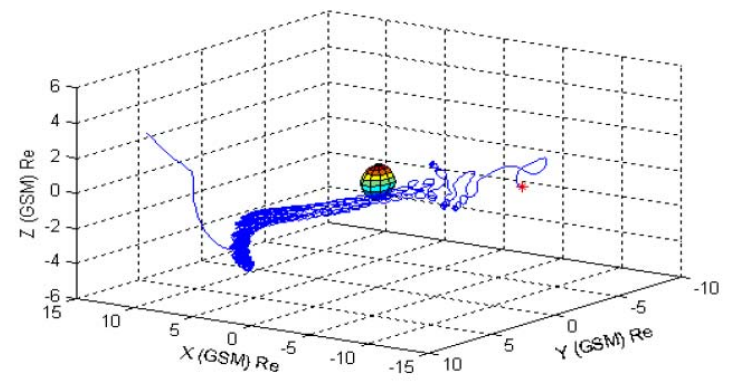

(b)

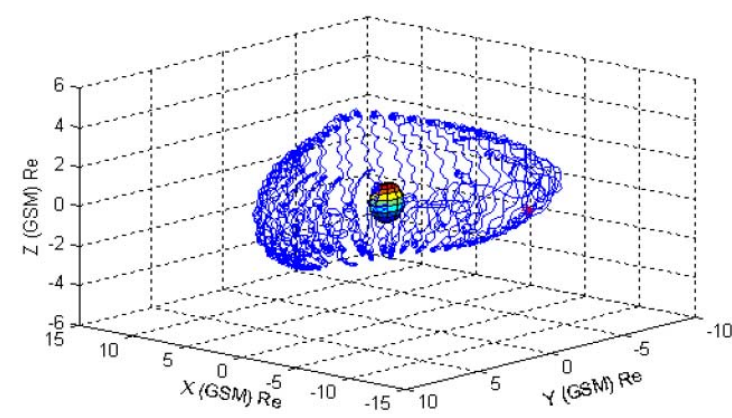

Fig. 10. Injection path of an $20 \mathrm{keV} \mathrm{O}^{+}$with equatorial pitch angle of $70^{\circ}$ initially located at $x=-8 R_{E}, y=-2 R_{E}$, and $z=0$ in the T96 $D_{s t}=-100$ magnetic field model. The upper (a) and lower (b) panels represent the cases without and with $E_{S}$, respectively.
On the other hand, the trajectory of $5 \mathrm{keV} \mathrm{O}^{+}$remains open even though the $E_{s}$ becomes nonzero. This implies that the higher the initial energy is of a particle, the easier its open drift path changes to closed. Similar phenomena can be seen for ions initially bouncing along the field lines. Figure 10 shows the injection path of an Oxygen ion initially located at $x=-8 R_{E}, y=-2 R_{E}$, and $z=0$, with energy $E=20 \mathrm{keV}$ and equatorial pitch angles $\alpha=70^{\circ}$. The adopted magnetic field and electric field models are T96 with $D_{s t}=-100 \mathrm{nT}$ and the Volland and Stern model, as well. The upper (a) and lower (b) panels represent the cases without and with the $E_{s}$, respectively. The 3-D trajectory plotted in Fig. 10b more clearly shows how an injected particle gradually becomes a symmetric ring current population under the action of the shielding field. A vast amount of calculations illustrate the same feature. All these strongly support our viewpoint that the shield electric field may play an important role in the formation of the symmetric ring current.

\section{Discussions and summary}

A study of ring current particle injection and intensification of the storm-time symmetric ring current is conducted in this paper. Three-dimensional TPTCs for Oxygen ions with different pitch angles are carried out, reflecting more accurately the process of ring current injection. 
There has been a symmetric ring current paradigm for years in which the ring current quickly becomes symmetric after the storm main phase, when ions drifting on open drift trajectories are trapped in closed drift paths, in response to the decrease in the convection electric field (Le et al., 2004). However, both the present work and Chen et al. (1994, 1997) challenge this paradigm that trapping of injected ions in closed paths may appear earlier in the main phase. Therefore, the peak in the symmetric ring current contribution happens later than the asymmetric ring current, but does not necessarily occur after the $D_{s t}$ minimum. The shielding electric field is created after the convection electric field increases for a period of time. This makes a number of injected particles change their drift paths and a great part of the partial ring current becomes the closed ring current.

In summary, the 3D-TPTC results show that: (a) when more realistic magnetic field models are adopted, injected ions often follow chaotic trajectories in non-adiabatic regions, which may have further implications in storm and ring current physics. (b) Shielding electric field $E_{S}$ may arise as a consequence of enhanced convection and co-exists with injection and $E_{C}$, which may cause the original open trajectories (in the case without $E_{S}$ ) to change into closed ones and play a role in the formation of the symmetric ring current. The shielding field mechanism is not in contradiction to the convection field fluctuation mechanism. Nevertheless, it is possible that the shielding field mechanism may manifest a more intrinsic factor in ring current injection than the convection field fluctuation mechanism. The present paper only illustrates qualitatively the importance of $E_{S}$ in the creation of the symmetric ring current. More accurate study invoking time-dependent $E_{C}$ and $E_{S}$ is highly desired.

Acknowledgements. This work is supported by NSFC project 40504017, 40390152, 40425004, 40528005 and 40374061, and Chinese Key Research Project No. 2006CB806300. We thank J. N. Tu for his great help in developing the 3-D TPTCs code.

Topical Editor I. A. Daglis thanks two referees for their help in evaluating this paper.

\section{References}

Chen, M. W., Schulz, M., and Lyons, L. R.: Simulations of phase space distributions of storm time proton ring current, J. Geophys. Res., 99, 5745-5759, 1994.

Chen, M. W., Schulz, M., and Lyons, L. R.: Modeling of ring current formation and decay: A review, Magnetic Storm, in: Magnetic Storm, Geophys. Monogr. Ser. Vol. 98, edited by: Tsurutani, B. T., Gonzalez, W. D., et al., AGU, Washington, D.C., 173-186, 1997.

Daglis, I. A.: The role of magnetosphere-ionosphere coupling in magnetic storm dynamics. Magnetic Storm, in: Magnetic Storm, Geophys. Monogr. Ser. Vol. 98, edited by: Tsurutani, B. T., Gonzalez, W. D., et al., AGU, Washington, D.C., 107-116, 1997.
Ebihara, Y., Fok, M.-C., Wolf, R. A., et al.: Influence of ionosphere conductivity on the ring current, J. Geophys. Res., 109, A08205, doi:10.1029/2003JA010351, 2004.

Fok, M.-C., Wolf, R. A., Spiro, R. W., and Moore, T. E.,: Comprehensive computational model of the Earth's ring current, J. Geophys. Res., 106, 8417-8424, 2001.

Fu, S. Y., Pu, Z. Y., Zong, Q. G., et al.: Ion composition variations in intense magnetic storms and their relation to storm evolution, Chinese, J. Geophys., 44, 1-6, 2001.

Fu, S. Y., Zong, Q. G., Wilken, B. et al.: Temporal and spatial variation of the ion composition in the ring current, in: Special Issue "Challenge to Long-standing Unsolved Space Physics Problems in the 20th Century", edited by: de Jager, C. and Akasofu, S., Space Sci. Rev., 95, 539-554, 2001.

Hasegawa, A. and Sato, T.: Generation of field aligned current during substorm, in: Dynamics of the Magnetosphere, edited by: Akasofu, S.-I. and Reidel, D., Norwell, Mass., 1979.

Heppner, J. P. and Maynard, N. C.: Empirical high-latitude electric field models, J. Geophys. Res., 92, 4467-4473, 1987.

Kamide, Y., McPherron, R. L., Gonzalez, W. D. et al.: Magnetic storms: Current understanding and outatanding questions, Magnetic Storm, in: Magnetic Storm, Geophys. Monogr. Ser. Vol. 98, edited by: Tsurutani, B. T., Gonzalez, W. D., et al., AGU, Washington, D.C., 1-19, 1997.

Kavanaugh, L. D., Jr., Freeman, J. W., Jr., and Chen, A. J.: Plasma flow in the magnetosphere, J. Geophys. Res., 73, 5511-5523, 1968.

Le, G., Russell, C. T., and Takahashi, K.: Morphology of the ring current derived from in-situ magnetic field measurements, Ann. Geophys., 22, 1267-1295, 2004,

http://www.ann-geophys.net/22/1267/2004/.

Liemohn M. W., Kozyra, J. U., Thomsen, M. F. et al.: Dominant role of the asymmetric ring current in producing the storm time Dst, J. Geophys. Res., 106, 10 883-10 904, 2001.

Lyons, L. R. and Williams, D. J.: Quantitative Aspects of Magnetospheric Physics, D. Reidel Publishing Company, Dordrecht, 1984.

Maynard, N. C. and Chen, A. J.: Isolated cold plasma regions: observations and their relation to possible production mechanisms, J. Geophys. Res., 80, 1009-1013, 1975.

McPherron, R. L.: Physical Processes Producing Magnetospheric Substorms and Storm, in" Geomagnetism Vol. 4, edited by: Jacobs, J.A., Academic Press, Harcourt Brace Jovanovich, Publishers, 1991.

Rowland, D. E. and Wygant, J. R.: Dependence of the large-scale, inner magnetospheric electric field on geomagnetic activity, J. Geophys. Res., 103, 14 959-14 964, 1998.

Sato T.: Auroral dynamics, in: Magnetospheric Plasma Physics, edited by: Nishida, A., Center for academic Publications, Tokyo, 1982.

Schield, M. A., Freeman, T. W., and Dessler, A. J.: A source for Field-Aligned Current at Auroral Latitudes, J. Geophys. Res., 74, 247-255, 1969.

Speiser, T. W.: Particle motion in the tail current sheet, Adv. Space Res., 11, 151-159, 1991.

Tsurutani, B. T. and Gonzalez, W. D.: The interplanetary causes of magnetic storms: A review, Magnetic Storm, in: Magnetic Storm, Geophys. Monogr. Ser. Vol. 98, edited by: Tsurutani, B. T., Gonzalez, W. D., et al., AGU, Washington, D.C., 77-89, 
1997.

Tsyganenko, N. A. and Stern, D. P.: Modeling the Global Magnetic Field of the Large-Scale Birkeland Current Systems, J. Geophys. Res., 101, 27 187-27 198, 1996.

Volland, H. A.: Model of the magnetospheric electric convection field, J. Geophys. Res., 83, 2695-1701, 1978.

Wolf, R. A.: Magnetospheric configuration, in: Introduction to Space Physics, edited by: Kivelson, M. G. and Russell, C. T., Cambridge University Press, 1995.

Wolf, R. A., Harel, M., Spiro, R. W., et al.: Computer Simulation of inner Magnetospheric Dynamics for the Magnetic Storm of 29 July 1977, J. Geophys. Res., 87, 5949-5961, 1982.
Wygant, J., Rowland, D., Singer, H. J., et al.: Experimental evidence on the role of the large spatial scale electric field in creating the ring current, J. Geophys. Res., 103, 29 527-29 544, 1998.

Xie, L., Pu, Z. Y., Yu, B., et al.: Injection of intense storm ring current ions, in: Solar-Terrestrial Magnetic Activity and Space Environment, COSPAR Colloquium Ser. Vol. 14, edited by: Wang, H. N. and Xu, R. L., COSPAR, Pergamon, 271-274, 2002.

Zhang. J. C., Tian, J. H., and Pu, Z. Y.: Interplanetory electric field and Dst index, Chinese, J. Space Sci., 21, 297-304, 2001. 